PREPARED FOR THE U.S. DEPARTMENT OF ENERGY, UNDER CONTRACT DE-AC02-76CH03073

PPPL-3769

PPPL-3769

UC-70

Nonlinear $\delta f$ Simulations of Collective Effects in Intense Charged Particle Beams

by

Hong Qin

January 2003

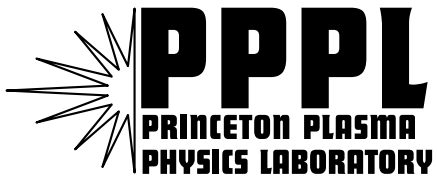

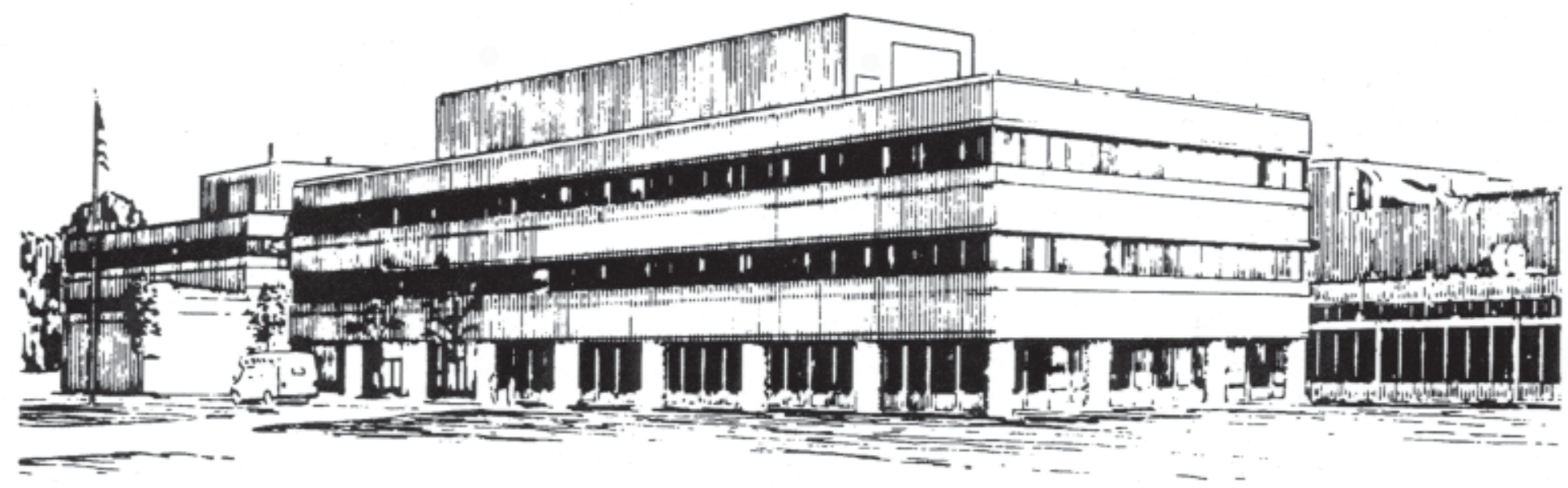

PRINCETON PLASMA PHYSICS LABORATORY PRINCETON UNIVERSITY, PRINCETON, NEW JERSEY 


\section{PPPL Reports Disclaimer}

This report was prepared as an account of work sponsored by an agency of the United States Government. Neither the United States Government nor any agency thereof, nor any of their employees, makes any warranty, express or implied, or assumes any legal liability or responsibility for the accuracy, completeness, or usefulness of any information, apparatus, product, or process disclosed, or represents that its use would not infringe privately owned rights. Reference herein to any specific commercial product, process, or service by trade name, trademark, manufacturer, or otherwise, does not necessarily constitute or imply its endorsement, recommendation, or favoring by the United States Government or any agency thereof. The views and opinions of authors expressed herein do not necessarily state or reflect those of the United States Government or any agency thereof.

\section{Availability}

This report is posted on the U.S. Department of Energy's Princeton Plasma Physics Laboratory Publications and Reports web site in Fiscal Year 2003. The home page for PPPL Reports and Publications is: http://www.pppl.gov/pub_report/

DOE and DOE Contractors can obtain copies of this report from:

U.S. Department of Energy

Office of Scientific and Technical Information

DOE Technical Information Services (DTIS)

P.O. Box 62

Oak Ridge, TN 37831

Telephone: (865) 576-8401

Fax: (865) 576-5728

Email: reports@adonis.osti.gov

This report is available to the general public from:

National Technical Information Service

U.S. Department of Commerce

5285 Port Royal Road

Springfield, VA 22161

Telephone: $1-800-553-6847$ or

(703) $605-6000$

Fax: (703) 321-8547

Internet: http://www.ntis.gov/ordering.htm 


\title{
Nonlinear $\delta$ f simulations of collective effects in intense charged particle beams
}

\author{
Hong Qin \\ Plasma Physics Laboratory, Princeton University, Princeton, NJ 08543, USA
}

\begin{abstract}
A nonlinear $\delta$ f particle simulation method based on the Vlasov-Maxwell equations has been recently developed to study collective processes in high-intensity beams, where space-charge and magnetic self-field effects play a critical role in determining the nonlinear beam dynamics. Implemented in the Beam Equilibrium, Stability and Transport (BEST) code [H. Qin, R. C. Davidson, and W. W. Lee, Phys. Rev. Special Topics on Accel. and Beams 3, 084401 (2000); 3, 109901 (2000).], the nonlinear $\delta$ f method provides a low-noise and self-consistent tool for simulating collective interactions and nonlinear dynamics of high-intensity beams in modern and next generation accelerators and storage rings, such as the Spallation Neutron Source and heavy ion fusion drivers. A wide range of linear eigenmodes of high intensity charged particle beams can be systematically studied using the BEST code. Simulation results for the electron-proton two-stream instability in the Proton Storage Ring experiment [R. Macek, et al, in Proc. of the Particle Accelerator Conference, Chicago, 2001 (IEEE, Piscataway, NJ, 2001), Vol. 1, p. 688.] at the Los Alamos National Laboratory agree well with experimental observations. Large-scale parallel simulations have also been carried out for the ion-electron two-stream instability in the very-high-intensity heavy ion beams envisioned for heavy ion fusion applications. In both cases, the simulation results indicate that the dominant two-stream instability has a dipole-mode (hose-like) structure and can be stabilized by a modest axial momentum spread of the beam particles.
\end{abstract}




\section{INTRODUCTION}

In contemporary periodic focusing accelerators and transport systems for applications such as spallation neutron sources, heavy ion fusion, and nuclear waste transmutation, the beam intensity has increased to regimes where collective processes and self-field effects play a significant role. It is therefore increasingly important to improve our theoretical understanding of the influence of the intense self-fields produced by the beam space charge and current on detailed equilibrium, stability and transport properties. A kinetic model based on the nonlinear Vlasov-Maxwell equations has been developed to study self-consistently the collective processes and self-field effects in high intensity charged particle beams [1, 2]. With the help of various analytical techniques, the kinetic approach has been successfully applied to a wide range of beam physics problem involving strong space-charge and high current. For example, a nonlinear kinetic stability theorem has been proved for charged particle beams with arbitrary space-charge intensity in the smooth focusing approximation [2-4]. However, many important questions are still unaccessible by analytical methods.

Recently, the $\delta$ f formalism, a low-noise, nonlinear perturbative particle simulation technique, has been developed to solve the nonlinear Vlasov-Maxwell equations for intense beam applications [5-9]. The $\delta$ f formalism has been implemented in the Beam Equilibrium, Stability and Transport (BEST) code at the Princeton Plasma Physics Laboratory. In addition, the BEST code is fully three-dimensional and has multi-species capability. It has been used to investigate the electron-ion two-stream instability [7, 9], temperature anisotropy instability [8], periodically-focusing beam propagation [7] and other collective processes. Especially, simulations carried out by the BEST code for the electron-proton (e-p) two-stream instability in the Proton Storage Ring (PSR) at the Los Alamos National Laboratory has produced results in good agreement with experimental observations.

In this paper, we will present a comprehensive description of the simulation capabilities of the BEST code and its applications to beam physics problems involving strong space charge and high current. The paper is organized as follows. The theoretical model and $\delta f$ formalism are outlined in Sec. II. Following a short description of the numerically solved equilibrium in Sec. III, we present in Sec. IV simulation results for two classes of collective modes, i.e., the body mode and the surface mode. In Sec. V, the electron-ion two-stream instability is studied in detail for a typical proton beam in the PSR experiment at moderate 
beam intensity, and for a Cesium beam with very high beam intensity for heavy ion fusion drivers. We summarize the conclusions and discuss future work in Sec. VI.

\section{THEORETICAL MODEL AND THE $\delta f$ FORMALISM FOR HIGH INTEN- SITY CHARGED PARTICLE BEAMS}

The theoretical model employed here to study the high intensity charged particle beams is based on the nonlinear Vlasov-Maxwell equations [1,2]. We consider a thin, continuous, high-intensity charged particle beam $(j=b)$ propagating in the $z$-direction. Possible background electron and ion components $(j=e, i)$ are allowed in the system. A background population of electrons, for example, can result by secondary emission when energetic ions or electrons strike the chamber wall, or through ionization of background neutral gas by the beam particles. Each charge component is described by a distribution function $f_{j}(\mathbf{x}, \mathbf{p}, t)$ $[1,2]$ in the phase space $(\mathbf{x}, \mathbf{p})$. The charge components $(j=b, e, i)$ propagate in the $z$-direction with characteristic axial momentum $\gamma_{j} m_{j} \beta_{j} c$, where $V_{j}=\beta_{j} c$ is the average directed axial velocity, $\gamma_{j}=\left(1-\beta_{j}^{2}\right)^{-1 / 2}$ is the relativistic mass factor, $e_{j}$ and $m_{j}$ are the charge and rest mass, respectively, of a $j$ 'th species particle, and $c$ is the speed of light in vacuo. While the nonlinear $\delta f$ formalism outlined here is readily adapted to the case of a periodic applied focusing field [10], for present purpose we make use of a smooth-focusing model in which the applied focusing force is described by $\mathbf{F}_{j}^{f o c}=-\gamma_{j} m_{j} \omega_{\beta j}^{2} \mathbf{x}_{\perp}$, where $\mathbf{x}_{\perp}=x \hat{\mathbf{e}}_{x}+y \hat{\mathbf{e}}_{y}$ is the transverse displacement of a particle from the beam axis, and $\omega_{\beta j}=$ const is the effective applied betatron frequency for transverse oscillations. Furthermore, in a frame of reference moving with axial velocity $\beta_{j} c$, the motion of a $j$ 'th species particle is assumed to be nonrelativistic. The space-charge intensity is allowed to be arbitrarily large, subject only to transverse confinement of each charge component. In a two-species system consisting of beam ions and stationary background electrons, for example, the beam ions are confined by the applied focusing force, while the background electrons are confined in the transverse plane by the space-charge potential $\phi(\mathbf{x}, t)$ produced by the excess ion charge. In the electrostatic and magnetostatic approximations, we represent the self-electric and self-magnetic fields as $\mathbf{E}^{s}=-\nabla \phi(\mathbf{x}, t)$ and $\mathbf{B}^{s}=\nabla \times A_{z}(\mathbf{x}, t) \hat{\mathbf{e}}_{z}$. The nonlinear Vlasov-Maxwell equations 
can be approximated by $[1,2]$

$$
\left\{\frac{\partial}{\partial t}+\mathbf{v} \cdot \frac{\partial}{\partial \mathbf{x}}-\left[\gamma_{j} m_{j} \omega_{\beta j}^{2} \mathbf{x}_{\perp}+e_{j}\left(\nabla \phi-\frac{v_{z}}{c} \nabla_{\perp} A_{z}\right)\right] \cdot \frac{\partial}{\partial \mathbf{p}}\right\} f_{j}(\mathbf{x}, \mathbf{p}, t)=0,
$$

and

$$
\begin{aligned}
\nabla^{2} \phi & =-4 \pi \sum_{j} e_{j} \int d^{3} p f_{j}(\mathbf{x}, \mathbf{p}, t), \\
\nabla^{2} A_{z} & =-\frac{4 \pi}{c} \sum_{j} e_{j} \int d^{3} p v_{z} f_{j}(\mathbf{x}, \mathbf{p}, t) .
\end{aligned}
$$

In the nonlinear $\delta$ f formalism [5-9], we divide the total distribution function into two parts, $f_{j}=f_{j 0}+\delta f_{j}$, where $f_{j 0}$ is a known equilibrium solution $(\partial / \partial t=0)$ to the nonlinear Vlasov-Maxwell equations (1) and (2), and the numerical simulation is carried out to determine the detailed nonlinear evolution of the perturbed distribution function $\delta f_{j}$. This is accomplished by advancing the weight function defined by $w_{j} \equiv \delta f_{j} / f_{j}$, together with the particles' positions and momenta. The equations of motion for the particles, obtained from the characteristics of the nonlinear Vlasov equation (1), are given by

$$
\begin{aligned}
\frac{d \mathbf{x}_{\perp j i}}{d t} & =\left(\gamma_{j} m_{j}\right)^{-1} \mathbf{p}_{\perp j i}, \\
\frac{d z_{j i}}{d t} & =v_{z j i}=\beta_{j} c+\gamma_{j}^{-3} m_{j}^{-1}\left(p_{z j i}-\gamma_{j} m_{j} \beta_{j} c\right), \\
\frac{d \mathbf{p}_{j i}}{d t} & =-\gamma_{j} m_{j} \omega_{\beta j}^{2} \mathbf{x}_{\perp j i}-e_{j}\left(\nabla \phi-\frac{v_{z j i}}{c} \nabla_{\perp} A_{z}\right) .
\end{aligned}
$$

Here the subscript " $j i$ " labels the $i$ 'th simulation particle of the $j$ 'th species. Furthermore, the dynamical equations for $w_{j i}$ is $[5,7-9]$

$$
\begin{aligned}
\frac{d w_{j i}}{d t} & =-\left(1-w_{j i}\right) \frac{1}{f_{j 0}} \frac{\partial f_{j 0}}{\partial \mathbf{p}} \cdot \delta\left(\frac{d \mathbf{p}_{j i}}{d t}\right), \\
\delta\left(\frac{d \mathbf{p}_{j i}}{d t}\right) & \equiv-e_{j}\left(\nabla \delta \phi-\frac{v_{z j i}}{c} \nabla_{\perp} \delta A_{z}\right),
\end{aligned}
$$

where $\delta \phi=\phi-\phi_{0}$ and $\delta A_{z}=A_{z}-A_{z 0}$. Here, the equilibrium solutions $\left(\phi_{0}, A_{z 0}, f_{j 0}\right)$ solve the steady-state $(\partial / \partial t=0)$ Vlasov-Maxwell equations (1) and (2). A wide variety of axisymmetric equilibrium solutions to Eqs. (1) and (2) have been investigated in the literature $[1,2,11]$. The perturbed distribution $\delta f_{j}$ is obtained through the weighted Klimontovich representation $[1,2]$

$$
\delta f_{j}=\frac{N_{j}}{N_{s j}} \sum_{i=1}^{N_{s j}} w_{j i} \delta\left(\mathbf{x}-\mathbf{x}_{j i}\right) \delta\left(\mathbf{p}-\mathbf{p}_{j i}\right)
$$


where $N_{j}$ is the total number of actual $j$ 'th species particles, and $N_{s j}$ is the total number of simulation particles for the $j$ 'th species. Maxwell's equations are also expressed in terms of the perturbed fields and perturbed density according to

$$
\nabla^{2} \delta \phi=-4 \pi \sum_{j} e_{j} \delta n_{j}, \quad \nabla^{2} \delta A_{z}=-\frac{4 \pi}{c} \sum_{j} \delta j_{z j}
$$

where

$$
\begin{aligned}
\delta n_{j} & =\frac{N_{j}}{N_{s j}} \sum_{i=1}^{N_{s j}} w_{j i} S\left(\mathbf{x}-\mathbf{x}_{j i}\right), \\
\delta j_{z j} & =\frac{e_{j} N_{j}}{N_{s j}} \sum_{i=1}^{N_{s j}} v_{z j i} w_{j i} S\left(\mathbf{x}-\mathbf{x}_{j i}\right) .
\end{aligned}
$$

Here, $S\left(\mathbf{x}-\mathbf{x}_{j i}\right)$ is a shape function distributing particles on the grids in configuration space $[7]$.

The nonlinear $\delta$ f particle simulations are carried out by iteratively advancing the particle motions, including the weights they carry, according to Eqs. (3) and (4), and updating the fields by solving the perturbed Maxwell's equations (6) with appropriate boundary conditions. Even though it is a perturbative approach, the $\delta \mathrm{f}$ method is fully nonlinear and simulates completely the original nonlinear Vlasov-Maxwell equations. Compared with conventional particle-in-cell (PIC) simulations, the noise level in $\delta$ f simulations is significantly reduced. The dominant numerical noise mechanisms in particle simulations, such as numerical collisions, are statistical. The $\delta$ f method reduces the noise level in the simulations because the statistical noise, which is of order $O\left(N_{s}^{-1 / 2}\right)$ for the total distribution function in the conventional particle-in-cell method, is only associated with the perturbed distribution function in the $\delta \mathrm{f}$ method. If the same number of simulation particles is used in the two approaches, then the noise level in the $\delta$ f method is reduced by a factor of $f / \delta f$ relative to the conventional PIC method. To achieve the same accuracy for the perturbed fields, the number of simulation particles used in the $\delta \mathrm{f}$ method is reduced by a factor of $(f / \delta f)^{2}$. For the e-p two-stream instability in the PSR experiment studied in Sec. V, we obtain satisfactory simulation results with about $10^{5}$ simulation particles using the $\delta \mathrm{f}$ method. If the conventional PIC method were used, then for a nonlinear saturation level of $1 \%$, about $10^{4}$ times more simulation particles would be needed to achieve the same accuracy. When studying the ion-electron two-stream instability in a high intensity heavy ion beam for heavy ion fusion drivers, it takes a relatively long time to simulate the instability due to the large mass 
ratio between the ions and the electrons $\left[m_{e} / m_{b}=1 /(1836 \times 133)=4.1 \times 10^{-6}\right.$, for cesium], and the fact that the growth rate of the instability is much smaller than the real frequency of the eigenmode. The low-noise $\delta f$ method is even more desirable in such applications.

The $\delta \mathrm{f}$ method can also be used to study detailed linear eigenmode and stability properties, provided the factor $\left(1-w_{j i}\right)$ in Eq. (4) is approximated by unity, and the forcing terms in Eq. (3) are replaced by the unperturbed force. Implementation of the 3D multispecies nonlinear $\delta \mathrm{f}$ simulation method described above is embodied in the BEST code [7-9] developed at the Princeton Plasma Physics Laboratory. The code advances the particle motions using a leap-frog or Runge-Kutta method, and solves Maxwell's equations in cylindrical geometry. For those fast particle motions which require much larger sampling frequency $1 / \Delta t$ than the frequency of the mode being studied, the code uses an adiabatic field pusher to advance the particles many time steps without solving for the perturbed fields. The upper limit for $\Delta t$, the time step to advance the particles' phase space position, is normally determined by the Courant condition. For the e-p two-stream instability, the electrons' transverse motion requires the smallest $\Delta t$, and the mode frequency is comparable to the electron bounce frequency in the transverse direction. We can therefore update the electrons' phase space positions more often than the field. On the IBM SP supercomputer at the National Energy Research Scientific Computing Center, the BEST code typically advances $4.2 \times 10^{11}$ particles $\times$ time-steps when simulating the electron-ion two-stream instability in high intensity beams.

\section{EQUILIBRIUM}

For practical accelerator experiments, it is important to obtain the equilibrium $(\partial / \partial t=0)$ distribution functions $f_{j 0}$ of the quiescent beam $(j=b)$ and any other possible charged particle components intentionally or unintentionally introduced into the system. From the equilibrium distribution function $f_{j 0}$, the rms radius, transverse emittance and other important beam parameters can be readily calculated. It is also necessary to know, analytically

or numerically, the equilibrium distribution function $f_{j 0}$ in order to carry out the particle simulations for the perturbed distribution function $\delta f_{j}$.

Although there are many possible choices of $f_{j 0}[1,2,11]$, in the present study we assume that the background equilibrium distribution $(\partial / \partial t=0)$ is the self-consistent bi-Maxwellian 
distribution with temperature $T_{j \perp}=$ const. in the $x-y$ plane, and temperature $T_{j \|}=$ const. in the $z$-direction. That is,

$$
\begin{aligned}
& f_{j 0}(r, \mathbf{p})=\frac{\hat{n}_{j}}{\left(2 \pi m_{j}\right)^{3 / 2} \gamma_{j}^{5 / 2} T_{j \perp} T_{j \|}^{1 / 2}} \\
& \times \exp \left\{-\frac{\left(p_{z}-\gamma_{j} m_{j} \beta_{j} c\right)^{2}}{2 \gamma_{j}^{3} m_{j} T_{j \|}}\right\} \\
& \times \exp \left\{-\frac{p_{\perp}^{2} / 2 \gamma_{j} m_{j}+\gamma_{j} m_{j} \omega_{\beta j}^{2} r^{2} / 2+e_{j}\left(\phi_{0}-\beta_{j} A_{z 0}\right)}{T_{j \perp}}\right\},
\end{aligned}
$$

where $\hat{n}_{j}$ is the density on axis $(r=0)$ of the $\mathrm{j}$ 'th species. Here, $\phi_{0}$ and $A_{z 0}$ are equilibrium self-field potentials, determined self-consistently from the nonlinear Maxwell equations

$$
\begin{gathered}
\frac{1}{r} \frac{\partial}{\partial r} r \frac{\partial \phi_{0}(r)}{\partial r}=-4 \pi \sum_{j} e_{j} \int d^{3} p f_{j 0}(r, \mathbf{p}), \\
\frac{1}{r} \frac{\partial}{\partial r} r \frac{\partial A_{z 0}(r)}{\partial r}=-\frac{4 \pi}{c} \sum_{j} e_{j} \int d^{3} p v_{z} f_{j 0}(r, \mathbf{p}) .
\end{gathered}
$$

Unlike the Kapchinskij-Vladimirskij (KV) distribution [1, 2], which is unstable due to the highly inverted distribution in phase space, a single-species charged particle beam with biMaxwellian distribution in Eq. 8 has been proven to be linearly and nonlinearly stable [2-4] for transverse perturbations with $k_{z}=0$. Numerically, Eqs. (8) and (9) can be easily solved. We demonstrate the equilibrium solutions using a set of beam parameters typical for heavy ion fusion drivers. We consider a $C s^{+}$beam with rest mass $m_{b}=133 m_{p}$, where $m_{p}$ is the proton rest mass, and kinetic energy $\left(\gamma_{b}-1\right) m c^{2}=2.5 \mathrm{GeV}$. To study the ion-electron twostream instability, a electron poplulation is introduced into the system with $V_{e}=0$ and $\omega_{\beta e}=$ 0 (corresponding to axially stationary electrons). The beam intensity is taken to be near the space-charge-dominated limit, corresponding to $s_{b} \equiv \hat{\omega}_{p b}^{2} / 2 \gamma_{b}^{2} \omega_{\beta b}^{2}=0.999$. The fractional charge neutralization $f \equiv \hat{n}_{e} / \hat{n}_{b}$ is taken to be $10 \%$, where $\hat{n}_{e}$ and $\hat{n}_{b}$ are the electron and beam ion densities on axis $(r=0)$. Plotted in Fig. 1 are the normalized equilibrium density profiles for the cesium ions and electrons, $n_{j}^{0}(r) / \hat{n}_{j}=\left(1 / \hat{n}_{j}\right) \int d^{3} p f_{j 0}(r, \mathbf{p}, t)(j=b, e)$, which are readily obtained once the equilibrium potentials $\phi_{0}$ and $A_{z 0}$ are solved numerically from Eqs. (8) and (9). The transverse temperatures of the electrons and ions in Fig. 1 are chosen to be $T_{b \perp} / \gamma_{b} m_{b} V_{b}^{2}=1.1 \times 10^{-6}$ and $T_{e \perp} / \gamma_{b} m_{b} V_{b}^{2}=2.47 \times 10^{-6}$, such that the ion and electron density profiles overlap radially. The overlapping of the electron density profile with that of the ions is expected to maximize the two-stream interaction and therefore the growth rate. In the space-charge-dominated limit $\left(s_{b} \rightarrow 1\right)$, if there is no electron population, the 


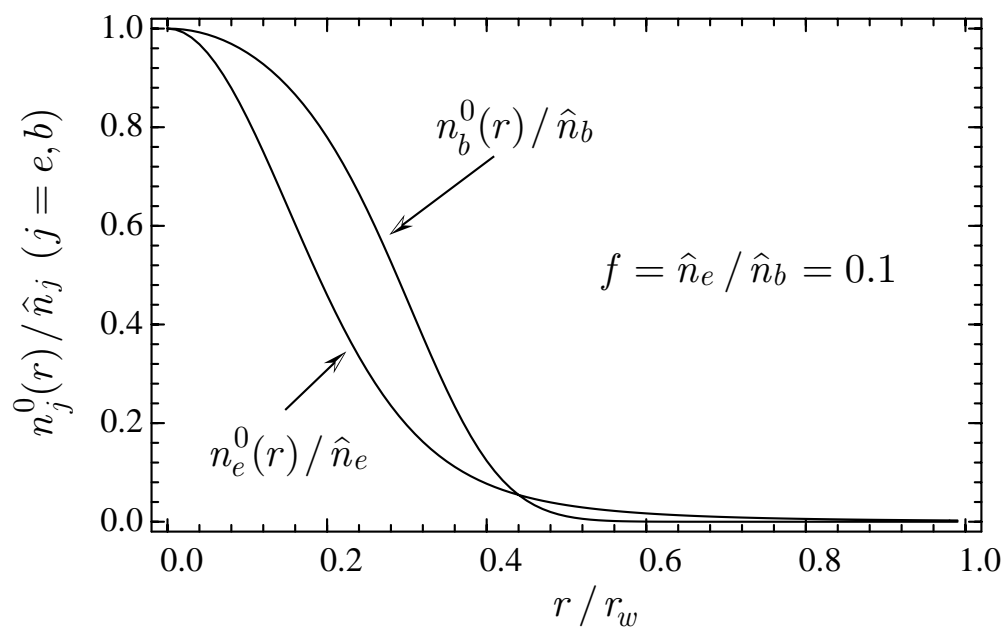

FIG. 1: Plots of the normalized density profiles of the equilibrium beam ions and background electrons.

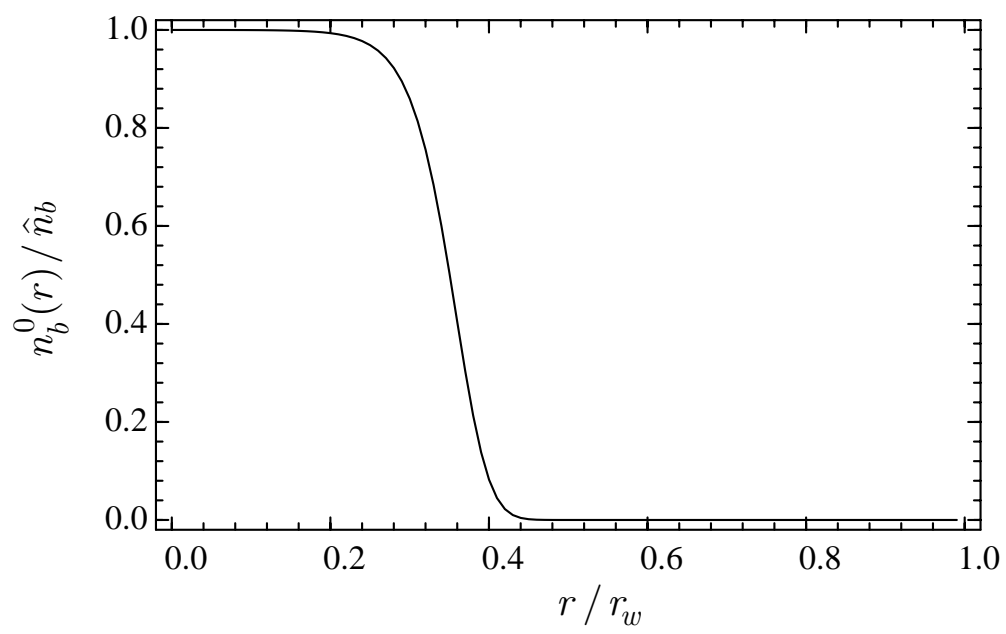

FIG. 2: Plots of the normalized density profile of the equilibrium beam ions with no background electron population.

beam will have a flat-top density profile as illustrated in Fig. 2. Plotted in Fig. 2 is the beam density profile with the same beam intensity parameter $s_{b}$ as the case in Fig. 1, but without a background electron population. Comparing the two cases, we conclude that the presence of a small population of background electrons offsets some of the space-charge force and produces the bell-shape beam density profile in Fig. 1. 


\section{EIGENMODE ANALYSIS IN HIGH INTENSITY BEAMS}

As mentioned in Sec. II, the $\delta$ f particle simulation method is an effective tool to study linear eigenmodes in a high intensity beam. In this section, we present two such examples. First, we simulate the $k_{z}=0, l=0(\partial / \partial z=\partial / \partial \theta=0)$ body mode for a $1.85 \mathrm{GeV}$ proton beam characteristic of the PSR experiment with axial current $I_{b}=N_{b} e_{b} \beta_{b} c=69 \mathrm{~A}$, directed axial velocity $V_{b}=0.84 c$, rms equilibrium beam radius $R_{b 0}=1.7 \mathrm{~cm}$, wall radius $r_{w}=5.0 \mathrm{~cm}$, normalized on-axis $(r=0)$ beam intensity $s_{b}=\hat{\omega}_{p b}^{2} / 2 \gamma_{b}^{2} \omega_{\beta b}^{2}=0.158$, and normalized beam temperature $T_{b} / \gamma_{b} m_{b} \beta_{b}^{2} c^{2}=7.22 \times 10^{-6}$. The system is perturbed about the equilibrium state by an initial density perturbation which varies smoothly across the beam radius, with zero net perturbed charge density. The evolution of the perturbation is followed from $t=0$ to $t=200 \omega_{\beta b}^{-1}$. Shown in Fig. 3(a) is the fast-Fourier-transform spectrum of the density perturbation at one spatial location, from which we can clearly identify the first four eigenmodes of the system at frequencies $\omega_{1}=1.94 \omega_{\beta b}, \omega_{2}=3.87 \omega_{\beta b}$, $\omega_{3}=5.83 \omega_{\beta b}$, and $\omega_{4}=7.77 \omega_{\beta b}$. The corresponding potential perturbation, $\delta \phi_{n}(r)$, for each eigenmode is plotted in Fig. 3(b). We follow the convention in previous analytical and numerical studies $[2,12]$, and use the notation $n=1,2,3, \ldots$ to label the radial mode number of the discrete eigenmodes. Numerically, $\delta \phi_{n}(r)$ is extracted from $\delta \phi(r, t)$ by determining the Fourier component of $\delta \phi(r, t)$ oscillating at frequency $\omega_{n}$. As is evident from Fig. 3, consistent with previous analytical and numerical studies, the eigenfunction $\delta \phi_{n}(r)$ has $n$ zeros when plotted as a function of $r$. Plotted in Fig. 4 is the dependence of the eigenfrequencies $\omega_{n}$ on the beam intensity parameter $s_{b}$ while the rms beam radius is kept constant. It is clear that starting from $\omega_{n}=2 n \omega_{\beta b}$ at $s_{b}=0$, the eigenfrequencies of the body modes decreases for increasing beam intensity.

As a second example, we study the linear surface mode for perturbations about a thermal equilibrium ion beam in the space-charge-dominated regime, with flat-top density profile. The BEST code, operating in its linear stability mode, has recovered very well-defined eigenmodes with mode structures and eigenfrequencies which agree well with theoretical predications $[2,13]$. For the dipole mode with azimuthal mode number $l=1$, the dispersion relation for these modes is given by[13]

$$
\omega=k_{z} V_{b} \pm \frac{\hat{\omega}_{p b}}{\sqrt{2} \gamma_{b}} \sqrt{1-\frac{r_{b}^{2}}{r_{w}^{2}}}
$$



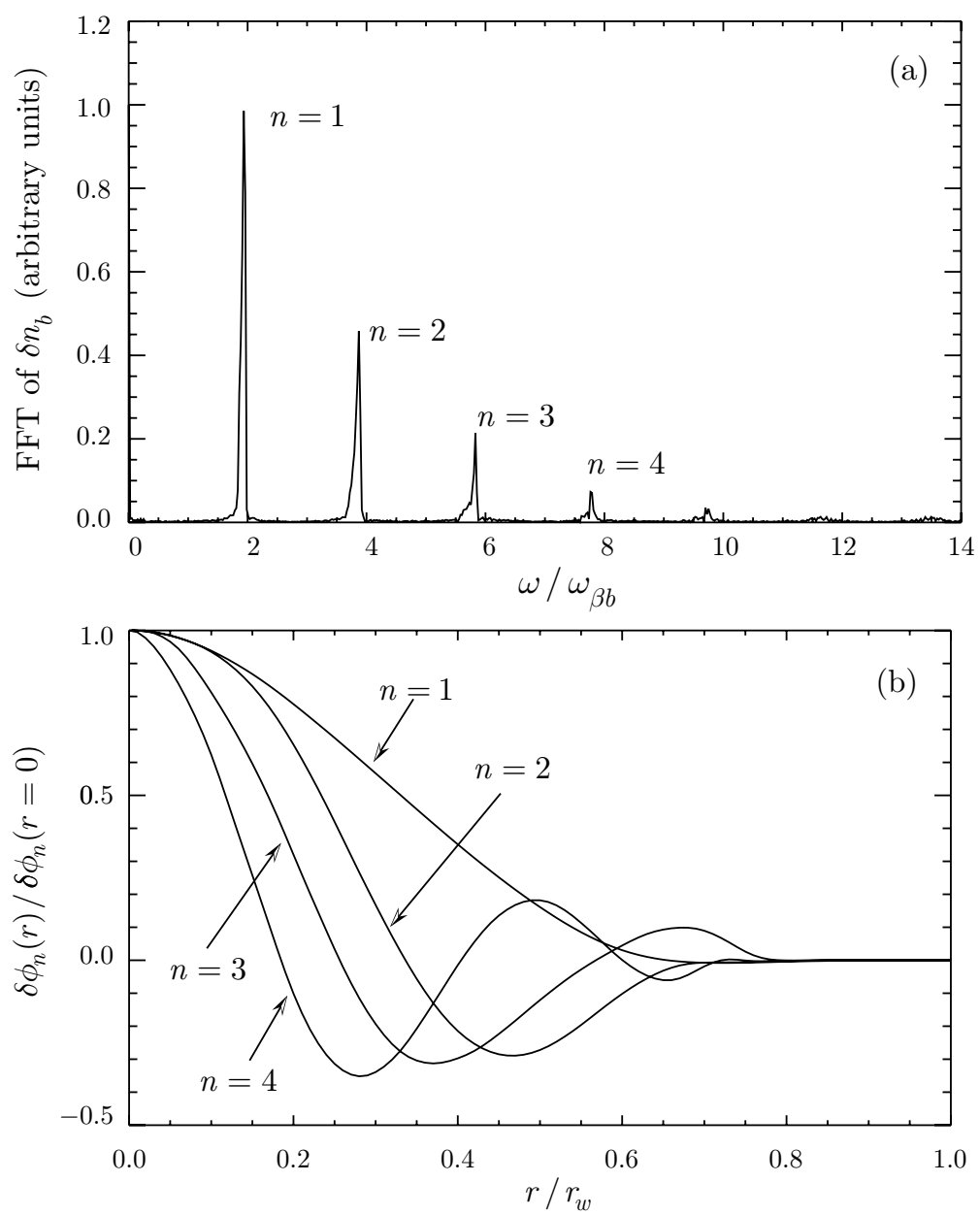

FIG. 3: (a)Fast-Fourier-transform (FFT) spectrum of perturbed density measured in the simulation, and (b) the corresponding eigenmode structures.

where $r_{b}$ is the radius of the beam edge, and $r_{w}$ is location of the conducting wall. In Eq. (10), $\hat{\omega}_{p b}^{2}=4 \pi \hat{n}_{b} e_{b}^{2} / \gamma_{b} m_{b}$ is the ion plasma frequency-squared, and $\hat{\omega}_{p b} / \sqrt{2} \gamma_{b} \simeq \omega_{\beta b}$ has been assumed in the space-charge-dominated limit. Shown in Fig. 5(a) is a comparison between plots of the eigenfrequency versus $r_{w} / r_{b}$ obtained from the simulations (diamonds and triangles) and that predicted by Eq. (10) (solid curves). The system parameters for this simulation are chosen close to the space-charge limit, and the perturbation has normalized axial wavenumber $k_{z} V_{b} / \omega_{\beta b}=2 \pi$. It is clear from Fig. 5 that the simulation results agree very well with theoretical predictions. The surface modes are of practical interest because they can be destabilized by a two-stream electron-ion interaction when background electrons are present. 


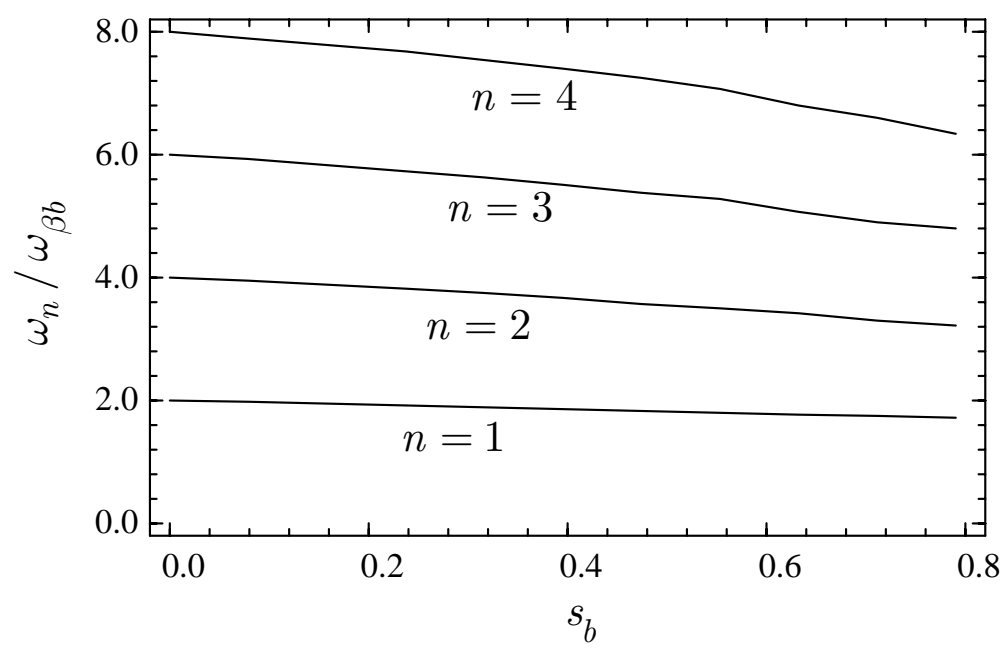

FIG. 4: Dependence of the eigenfrequencies on the beam intensity parameter $s_{b}$.

\section{TWO-STREAM INSTABILITY}

It has been recognized recently, both in theoretical studies and in experimental observations [2, 13-25], that the relative streaming motion of the high-intensity beam particles through a background charge species provides the free energy to drive the classical twostream instability, appropriately modified to include the effects of de space charge, relativistic kinematics, presence of a conducting wall, etc. A well-documented example is the e-p instability observed in the PSR experiment [11-12], although a similar instability also exists for other ion species, including ion-electron interactions in electron storage rings [13-15]. When electrons are present, two-stream interactions in heavy ion fusion drivers are expected to be stronger than the two-stream instabilities observed so far in proton machines (as well as electron machines) because of the much larger beam intensity. As an example of using the BEST code to study beam instabilities, we present here the simulation results of the ion-electron two-stream instabilities in a typical heavy ion fusion driver and in the PSR experiment.

For the case of a typical heavy ion fusion driver, we consider a $2.5 \mathrm{GeV} \mathrm{Cs}{ }^{+}$beam carrying 3000 A current propagating in a cylindrical chamber with wall radius $r_{w}=9 \mathrm{~cm}$. The beam intensity is taken to be near the space-charge-dominated limit, corresponding to $s_{b}=0.999$. The fractional charge neutralization $f \equiv \hat{n}_{e} / \hat{n}_{b}$ is taken to be $10 \%$.The ion-electron equilibrium solution is plotted in Fig. 1. After small-amplitude perturbations 

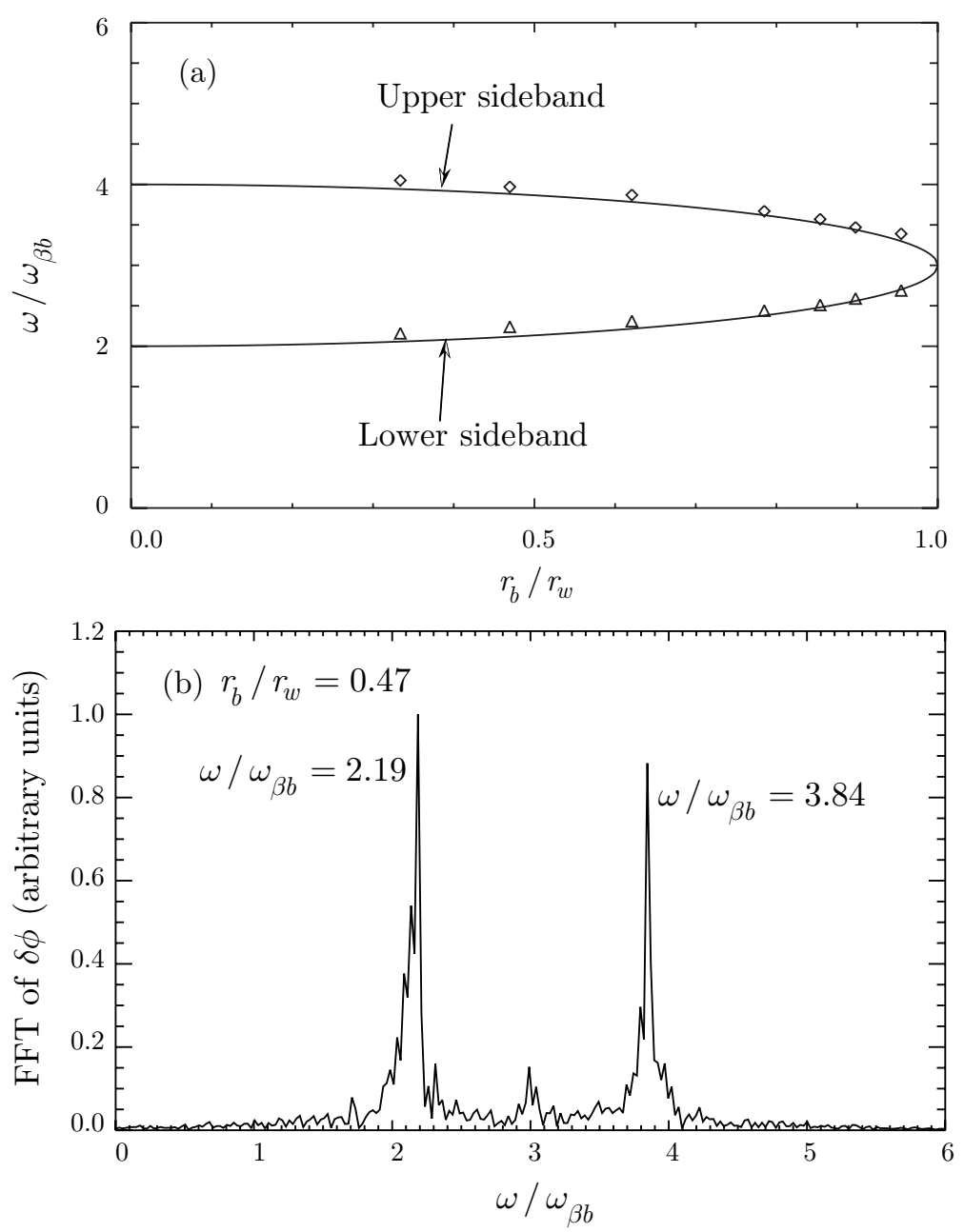

FIG. 5: (a) Measured dipole-mode oscillation frequency versus $r_{b} / r_{w} \simeq \sqrt{2} R_{b 0} / r_{w}$, and (b) FastFourier-transform spectrum for fixed value of $r_{b} / r_{w}=0.47$.

are excited at $t=0$, the system is evolved self-consistently for thousands of oscillation periods. Plotted in Fig. 6 is the time history of the beam density perturbation at one spatial location. Evidently, after an initial transition period, the perturbation grows exponentially, which is the expected behavior of an instability during the linear growth phase. In Fig. 7, the $x-y$ projection of the perturbed potential $\delta \phi$ at a fixed longitudinal position are plotted at $t=0$ and $t=32.5 / \omega_{\beta b}$. Clearly, $\delta \phi$ grows to a moderate amplitude by $t=3.25 / \omega_{\beta b}$, and the $l=1$ dipole mode is the dominant unstable mode, for which the growth rate is measured to be $\operatorname{Im} \omega=0.78 \omega_{\beta b}$. The $l=1$ dipole-mode instability observed here has features similar to the hose instability [26] in the collisionless limit. The real eigenfrequency of the mode is $\operatorname{Re} \omega=480 \omega_{\beta b}$, and the normalized wavelength at maximum growth is 


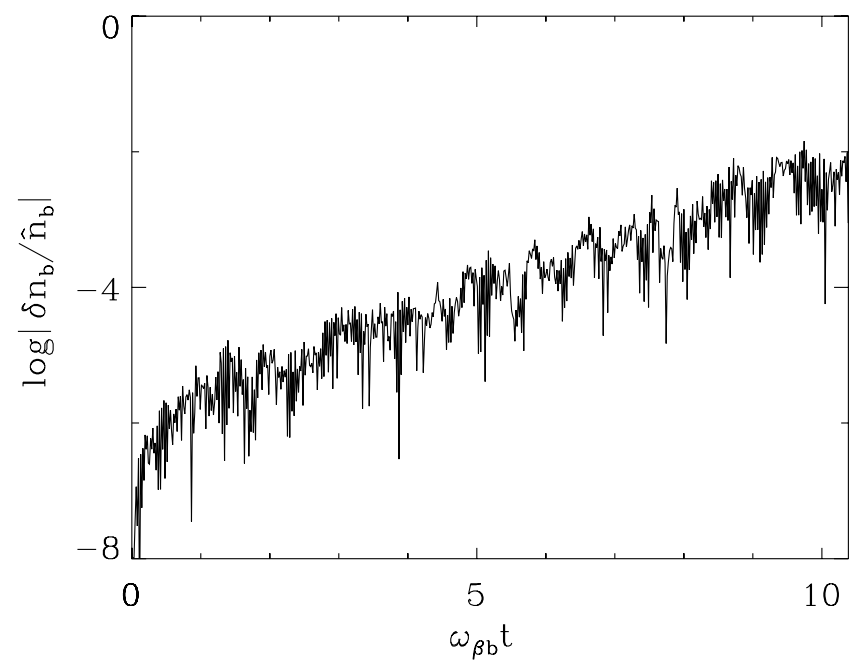

FIG. 6: Time history of perturbed density $\delta n_{b} / \hat{n}_{b}$ at a fixed spatial location. After an initial transition period, the $l=1$ dipole-mode perturbation grows exponentially.

$k_{z} V_{b} / \omega_{\beta b}=480.4$. Plots of the instability growth rate $\operatorname{Im} \omega$ versus $k_{z} V_{b} / \omega_{\beta b}$, with other parameters kept constant, are shown in Fig. 8. The $k_{z} V_{b} / \omega_{\beta b}$ dependence of the growth rate is qualitatively consistent with the analytical results obtained for uniform-density beams [13]. The important physics here is that only for a certain range of $k_{z} V_{b} / \omega_{\beta b}$ can the collective mode of the beam ions effectively resonate with the electrons and produce instability.

In the simulation results presented above, we have assumed initially cold beam ions in the longitudinal direction $\left(\Delta p_{b \|} / p_{b \|}=0\right)$ to maximize the growth rate of the instability. Here, $p_{b \|}=\gamma_{b} m_{b} V_{b}$. In general, when the longitudinal momentum spread of the beam ions is finite, Landau damping by parallel ion kinetic effects provides a mechanism that reduces the growth rate. Shown in Fig. 9 is a plot of the maximum linear growth rate $(\operatorname{Im} \omega)_{\max }$ versus the normalized initial axial momentum spread $\Delta p_{b \|} / p_{b \|}$ obtained in the numerical simulations. As evident from Fig. 9 , the growth rate decreases dramatically as $\Delta p_{b \|} / p_{b \|}$ is increased. When $\Delta p_{b \|} / p_{b \|}$ is high enough, about $0.58 \%$ for the case in Fig. 9 , the mode is completely stabilized by longitudinal Landau damping effects by the beam ions. This result agrees qualitatively with theoretical predications [15]. For a fixed value of $\Delta p_{b \|} / p_{b \|}$, the growth rate obtained from the simulation is several times smaller than the theoretical value $[2,13-15]$. This difference can be attributed to the fact that the theoretical value 

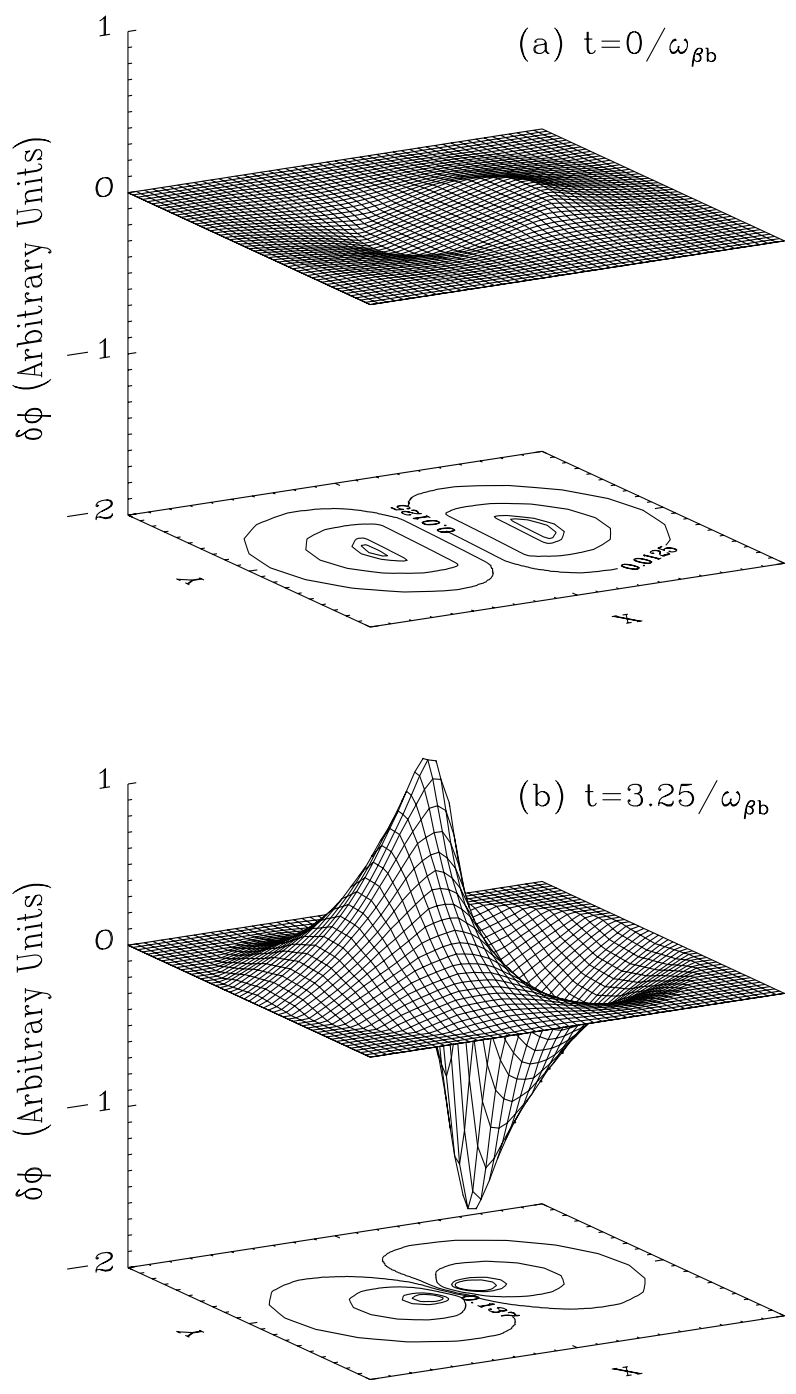

FIG. 7: The $x-y$ projection (at fixed value of $z$ ) of the perturbed electrostatic potential $\delta \phi(x, y, t)$ for the ion-electron two-stream instability growing from a small initial perturbation, shown at (a) $t=0$, and (b) $\omega_{\beta b} t=3.25$.

is derived for a Kapchinskij-Vladimirskij (KV) beam with flat-top density profile, whereas the simulations are carried out for a more realistic thermal equilibrium beam with bellshape density profile. Because the phase velocity of the unstable mode in the longitudinal direction is far removed from the electron velocity distribution $\left|\omega / k_{z}\right| \gg V_{e}+v_{T e \|}$, we do not expect the longitudinal electron temperature to significantly affect the growth rate of the instability. The nonlinear space-charge potential due to the bell-shape density profiles 


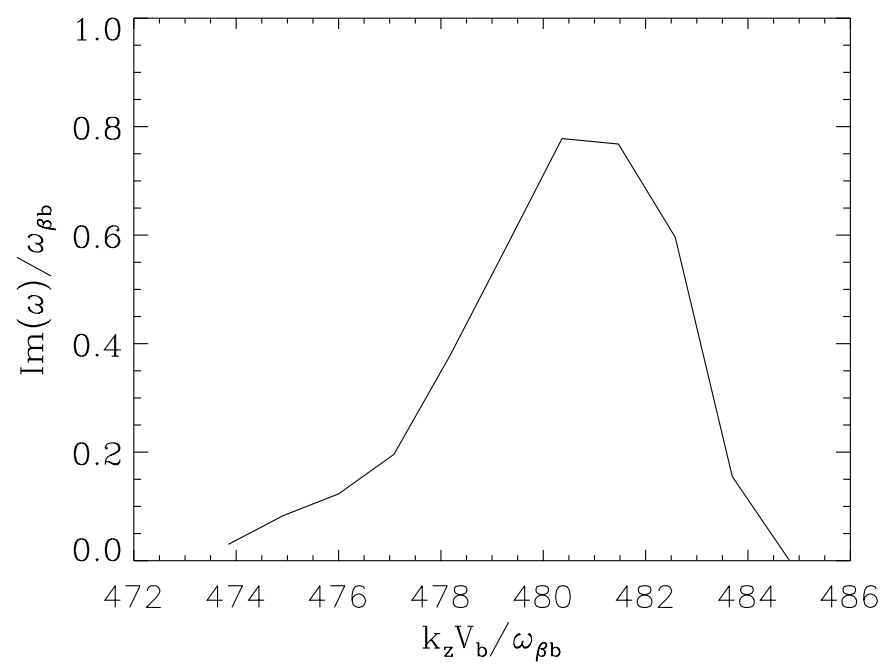

FIG. 8: Plot of the linear growth rate versus $k_{z} V_{b} / \omega_{\beta b}$.

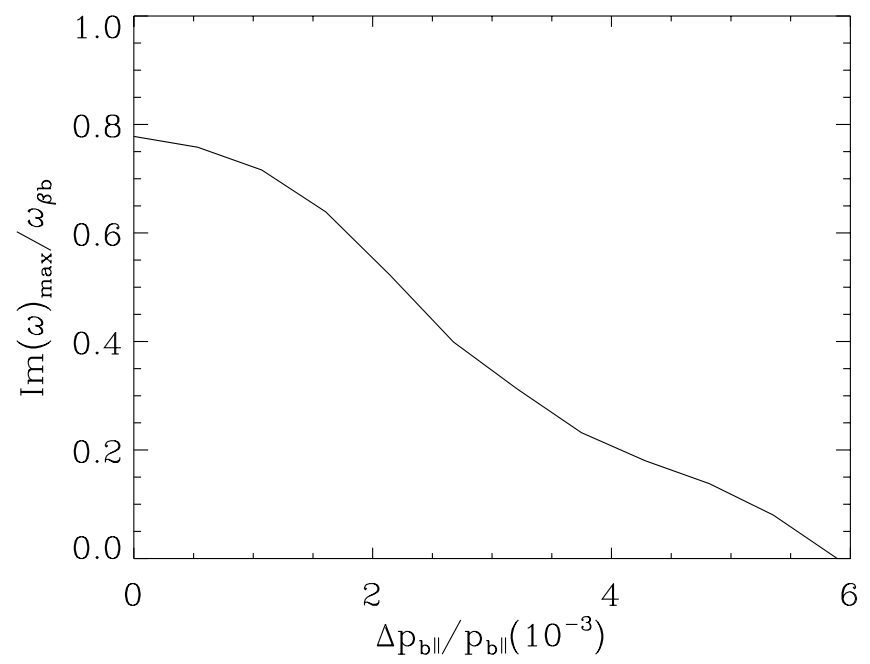

FIG. 9: The maximum linear growth rate $(\operatorname{Im} \omega)_{\max }$ of the ion-electron two-stream instability decreases as the longitudinal momentum spread of the beam ions increases.

induces substantial tune spread in the transverse direction, which provides a growth rate reduction mechanism for the two-stream instability.

Similar simulations have been carried out for the e-p two-stream instability in the PSR experiment, even though the numerical values of the key parameters of the instability, such as the growth rate and the real frequency, differs by several orders of magnitude compared 


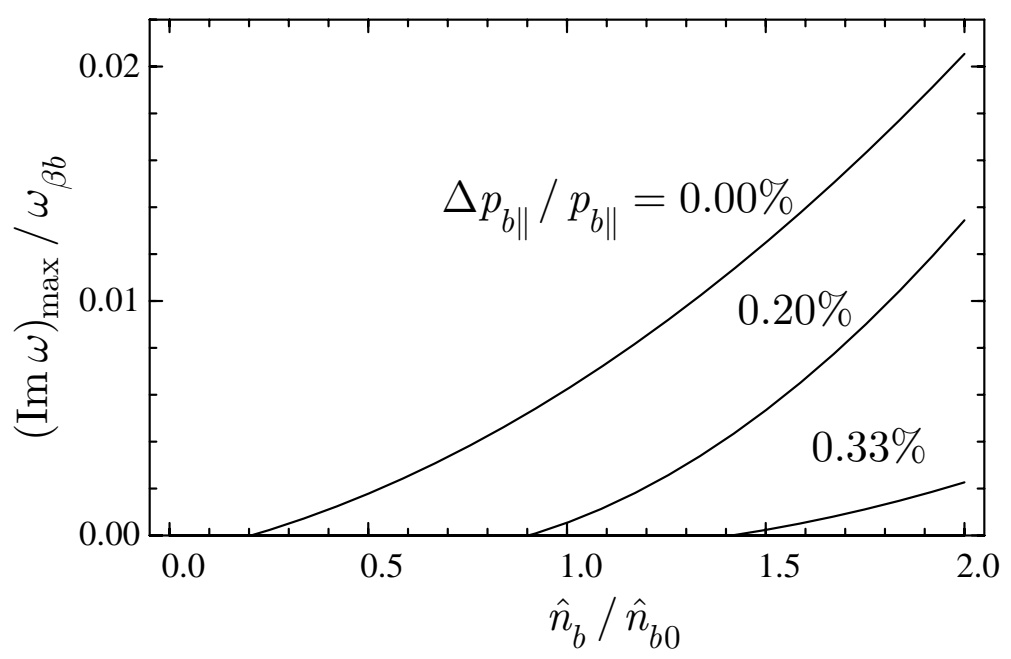

FIG. 10: Maximum growth rate versus normalized beam density for different values of initial axial momentum spread of the beam ions and fractional charge neutralization $f=\hat{n}_{e} / \hat{n}_{b}=0.08$.

with the ion-electron instability in a heavy ion fusion driver. In the parameter regime of the PSR experiment, the simulation results agree well with the experimental observations in terms of the real frequency, wavelength and mode structure [7]. For brevity, we present here only the threshold properties of the instability. Detailed numerical investigations of the e-p instability have been carried out for a wide range of beam intensities and fractional charge neutralization. The space-charge intensity varies from moderate to strong, corresponding to $0.008 \leq s_{b}=\hat{\omega}_{p b}^{2} / 2 \gamma_{b}^{2} \omega_{\beta b}^{2} \leq 0.158$, where $\hat{\omega}_{p b}^{2}=4 \pi \hat{n}_{b} e_{b}^{2} / \gamma_{b} m_{b}$ is the on-axis $(r=0)$ ion plasma frequency-squared. The fractional charge neutralization $f \equiv \hat{n}_{e} / \hat{n}_{b}$ is allowed to vary from $5 \%$ to $25 \%$, where $\hat{n}_{e}$ and $\hat{n}_{b}$ are the electron and beam ion number densities on axis $(r=0)$. In Fig. 10, for the case where $f=\hat{n}_{e} / \hat{n}_{b}=0.08$, the maximum growth rate in the simulations is plotted versus the normalized beam density $\hat{n}_{b} / \hat{n}_{b 0}$ for different values of initial axial momentum spread. Here, $\hat{n}_{b 0}=9.41 \times 10^{8} \mathrm{~cm}^{-3}$ is the beam ion number density on axis for the baseline case, corresponds to $\hat{\omega}_{p b}^{2} / 2 \gamma_{b}^{2} \omega_{\beta b}^{2}=0.079$ and an average current of $35 \mathrm{~A}$ in the PSR experiment. It is evident from the results shown in Fig. 10 that the growth rate is an increasing function of normalized beam density $\hat{n}_{b} / \hat{n}_{b 0}$, but a decreasing function of the longitudinal momentum spread, which qualitatively agrees with previous analytical results [15]. As demonstrated in Fig. 9, a larger longitudinal momentum spread induces stronger Landau damping by parallel kinetic effects and therefore reduces the growth rate of the instability, whereas higher beam intensity provides more free energy to drive a stronger 
instability.

As a result of the presence of several important damping mechanisms, an instability threshold is observed in the simulations. Plotted in Fig. 11 is the instability threshold in terms of the normalized beam density $\hat{n}_{b} / \hat{n}_{b 0}$ as a function of momentum spread $\Delta p_{b \|} / p_{b \|}$ for different values of fractional charge neutralization $f$. Evidently, larger momentum spread and smaller fractional charge neutralization imply a higher density threshold for the instability to occur. For a specified value of $f$, if $\left(\Delta p_{b \|} / p_{b \|}, \hat{n}_{b} / \hat{n}_{b 0}\right)$ fall below the curves in Fig. 11, then there is no two-stream instability. Finally, in Fig. 12, we simulate an unstable case to its fully

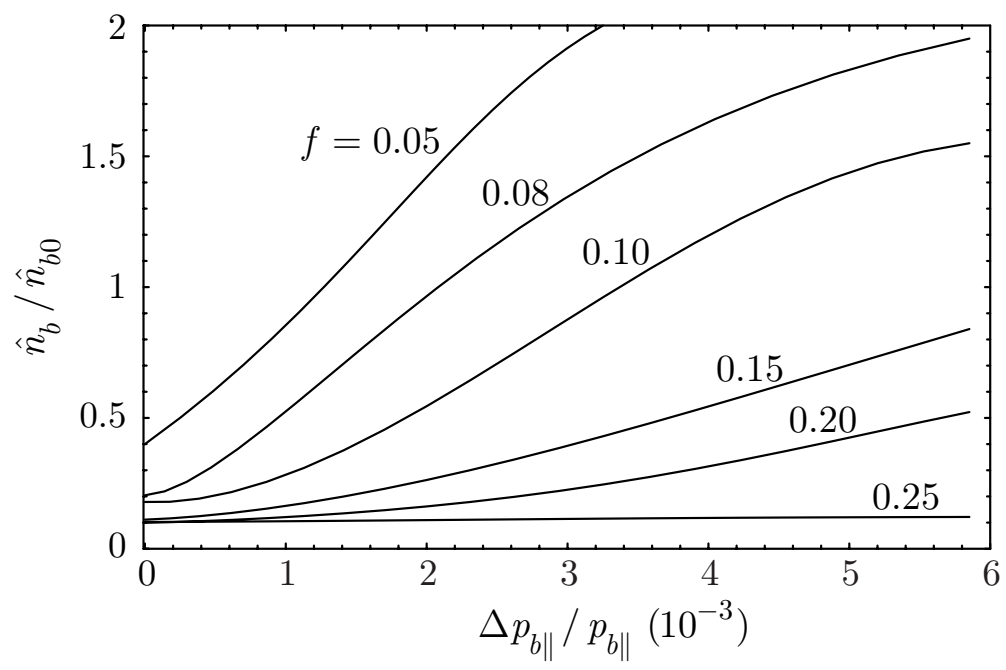

FIG. 11: Density threshold for the two-stream instability as a function of beam axial momentum spread for different values of fractional charge neutralization.

nonlinear phase. This case corresponds to $\hat{n}_{b} / \hat{n}_{b 0}=1, \hat{\omega}_{p b}^{2} / 2 \gamma_{b}^{2} \omega_{\beta b}^{2}=0.079, f=0.1$, and $\Delta p_{b \|}=0=\Delta p_{e \|}$ at $t=0$. In Fig. 12, the time history of the density perturbations at fixed spatial location is shown for both species. There are basically two phases for the evolution of the instability. The first phase is the linear stage where the density perturbations for both species grow exponentially. However, due to the large mass ratio between the protons and the electrons, the density perturbation amplitude for the electrons is much larger than that for the protons. When the linear growth saturates, the saturation level for the electron density perturbation is therefore much larger. The saturation level for the electron density perturbation shown in Fig. 12 is about 8\%, whereas the saturation level for the proton density is very small (less than $0.1 \%$ ). 

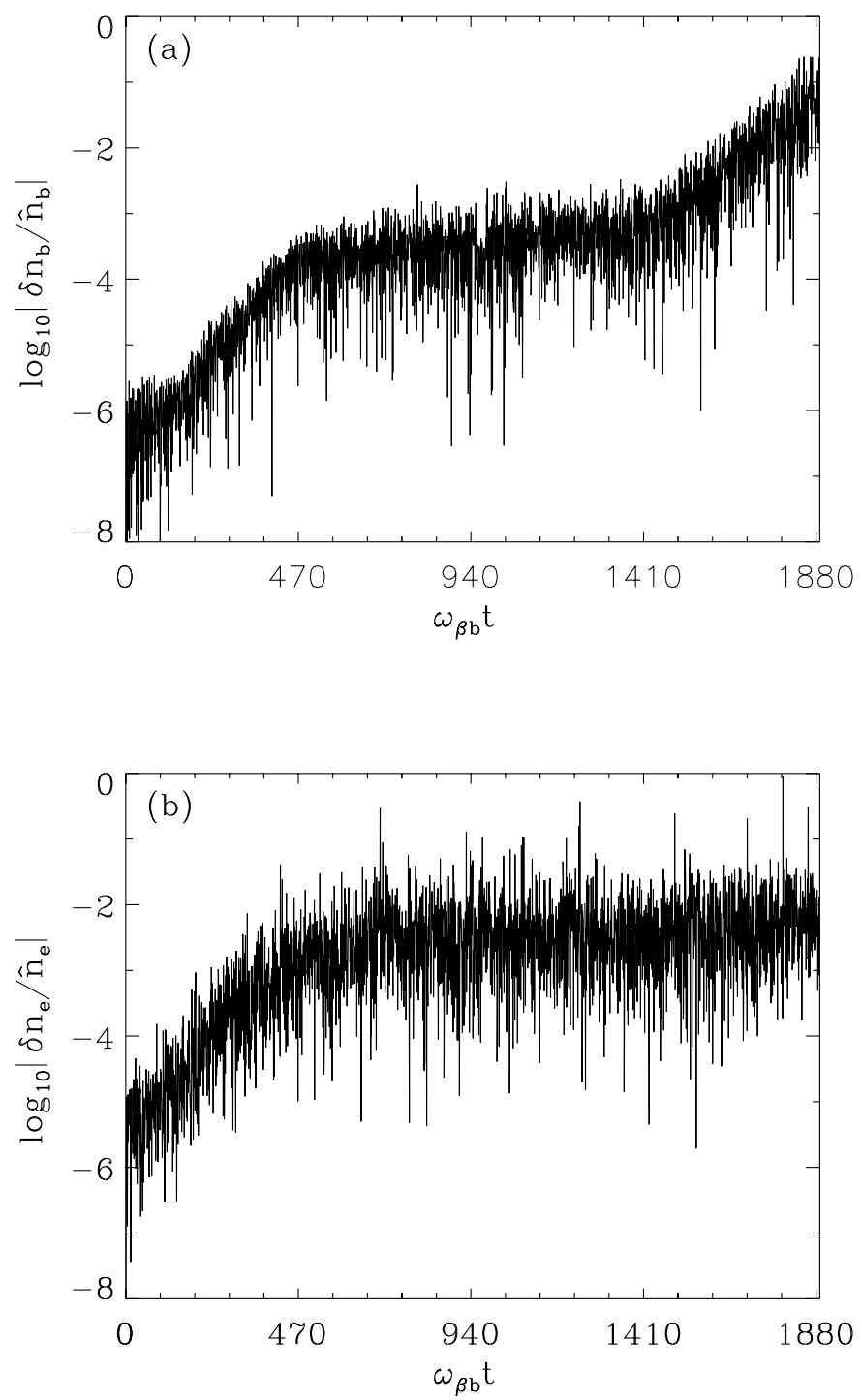

FIG. 12: Linear and nonlinear phases of the e-p two-stream instability. Plotted is the time history of the density perturbation for the (a) protons and (b) electrons at a fixed spatial location.

The second phase of the instability is the nonlinear phase, starting approximately at $t=500 / \omega_{\beta b}$, during which the electron density perturbation level stays nearly constant around the $8 \%$ level. In this phase, the electron density perturbation shows no extra dynamical behavior other than the initial nonlinear saturation. However, the proton density perturbation grows first slowly and then very fast after $t=1400 / \omega_{\beta b}$ to a high level, considerably larger than that of the electron density perturbation. This simulation result suggests 
that the late-time growth of the e-p instability observed experimentally in PSR has likely passed the initial linear growth and saturation phase, and entered the second stage of strong nonlinear growth evident in Fig. 12. It also points to the possible physical mechanism proposed by Channell [27] that due to the large mass ratio, the electron density perturbation quickly saturates long before the proton density perturbation becomes sizeable, and the large electron density fluctuation level then provides a newly developed background force that drives the proton density perturbations to a large level on a longer time scale [27].

\section{CONCLUSIONS}

In conclusion, a 3D multispecies nonlinear $\delta$ f particle simulation method has been developed to study collective processes in intense charged particle beams described selfconsistently by the Vlasov-Maxwell equations. Compared with conventional particle-in-cell simulations, the noise level in nonlinear perturbative particle simulations is significantly reduced. Implemented in the BEST code, the $\delta f$ formalism has been tested and applied in different beam parameter regimes. The code can be easily switched between linear and nonlinear operation, and used to study both linear stability properties and nonlinear beam dynamics. Linear eigenmodes of high intensity charged particle beams, such as the body modes and the surface modes, have been systematically studied using the BEST code. In particular, large-scale parallel simulations have also been carried out to study the ion-electron two-stream instability in the very-high-intensity heavy ion beams envisioned for heavy ion fusion applications, and for the e-p two-stream instability observed in the PSR experiment. Important properties of this instability were investigated numerically, and are found to be in qualitative agreement with theoretical predictions and the PSR experiment. Numerically, the instability threshold was found to decrease with increasing fractional charge neutralization, and increase with increasing axial momentum spread of the beam particles. In the nonlinear phase, the simulation results showed that the instability first saturates at a relatively low level, and subsequently grows to a much larger level. Even though a wide range

of collective effects in high intensity charged particle beams have been studied, the present simulations have been curried out for a long coasting beam. Investigations of the effects of finite bunch length, inclusion of electron production mechanisms, and other extensions have been planned for future work. 


\section{Acknowledgments}

This research was supported by the Department of Energy. The author thanks Ronald

C. Davidson, Edward A. Startsev and W. Wei-li Lee for many productive discussions and comments.

[1] R. C. Davidson, Physics of Nonneutral Plasmas (Addison-Wesley Publishing Co., 1990, reissued by World Scientific, 2001).

[2] R. C. Davidson and H. Qin, Physics of Intense Charged Particle Beams in High Energy Accelerators (World Scientific, 2001).

[3] R. C. Davidson, Physics of Plasmas 5, 3459 (1998).

[4] R. C. Davidson, Physical Review Letters 81, 991 (1998).

[5] W. W. Lee, Q. Qian, and R. C. Davidson, Phys. Lett. A 230, 347 (1997); Q. Qian, W. W. Lee, and R. C. Davidson, Phys. Plasmas 4, 1915 (1997).

[6] P. H. Stoltz, R. C. Davidson, and W. W. Lee, Phys. Plasmas 6, 298 (1999).

[7] H. Qin, R. C. Davidson, and W. W. Lee, Phys. Rev. Special Topics on Accel. and Beams 3, 084401 (2000); 3, 109901 (2000).

[8] E. A. Startsev, R. C. Davidson, and H. Qin, Phys. Plasmas 9, 3138 (2002).

[9] H. Qin, R. C. Davidson, W. W. Lee, and E. A. Startsev, in Proc. of the Particle Accelerator Conference, Chicago, 2001 (IEEE, Piscataway, NJ, 2001) Vol. 1, p. 693 .

[10] R. C. Davidson, H. Qin, and P. J. Channell, Phys. Rev. Special Topics on Accel. and Beams 2, 074401 (1999); 3, 029901 (2000); P. J. Channell, Phys. Plasmas 6, 982 (1999).

[11] R. C. Davidson, H. Qin and S. Lund, "Kinetic description of intense beam propagation through a periodic focusing field for uniform phase-space density", submitted to Phys. Rev. Special Topics on Accel. and Beams (2002).

[12] S. M. Lund and R. C. Davidson, Phys. Plasmas 5, 3028 (1998)

[13] R. C. Davidson, H. Qin, P. H. Stoltz, and T. -S. Wang, Phys. Rev. Special Topics on Accel. and Beams 2, 054401 (1999), and references therein.

[14] R. C. Davidson, H. Qin, and T. -S. Wang, Phys. Lett. A 252, 213 (1999).

[15] R. C. Davidson and H. Qin, Phys. Lett. A 270, 177 (1999). 
[16] T.-S. Wang, P. J. Channell, R. J. Macek, R. C. Davidson, in Proc. of the Particle Accelerator Conference, Chicago, 2001 (IEEE, Piscataway, NJ, 2001), Vol. 1, p. 704.

[17] D. G. Koshkarev and P. R. Zenkevich, Particle Accel. 3, 1 (1972).

[18] E. Keil and B. Zotter, CERN Report CERN-ISR-TH/71-58 (1971).

[19] L. J. Laslett, A. M. Sessler, and D. Möhl, Nucl. Instr. Meth. A 121, 517 (1974).

[20] D. Neuffer, E. Colton, D. Fitzgerald, T. Hardek, R. Hutson, R. Macek, M. Plum, H. Thiessen, and T. -S. Wang, Nucl. Instr. Meth. Phys. Res. A321, 1 (1992).

[21] R. J. Macek, in American Institute of Physics Conference Proceedings (American Institute of Physics, Melville, NY, 1998), Vol. 448, p. 116.

[22] R. Macek, et al, in Proc. of the Particle Accelerator Conference, Chicago, 2001 (IEEE, Piscataway, NJ, 2001), Vol. 1, p. 688.

[23] M. Izawa, Y. Sato, and T. Toyomasu, Phys. Rev. Lett. 74, 5044 (1995).

[24] J. Byrd, A. Chao, S. Heifets, M. Minty, T. O. Roubenheimer, J. Seeman, G. Stupakov, J. Thomson, and F. Zimmerman, Phys. Rev. Lett. 79, 79 (1997).

[25] K. Ohmi, Phys. Rev. E 55, 7550 (1997).

[26] E. P. Lee, Phys. Fluids 21, 1327 (1978).

[27] P. J. Channell, Phys. Rev. Special Topics on Accel. and Beams 5, 114401 (2002). 


\section{External Distribution}

Plasma Research Laboratory, Australian National University, Australia

Professor I.R. Jones, Flinders University, Australia

Professor João Canalle, Instituto de Fisica DEQ/IF - UERJ, Brazil

Mr. Gerson O. Ludwig, Instituto Nacional de Pesquisas, Brazil

Dr. P.H. Sakanaka, Instituto Fisica, Brazil

The Librarian, Culham Laboratory, England

Mrs. S.A. Hutchinson, JET Library, England

Professor M.N. Bussac, Ecole Polytechnique, France

Librarian, Max-Planck-Institut für Plasmaphysik, Germany

Jolan Moldvai, Reports Library, MTA KFKI-ATKI, Hungary

Dr. P. Kaw, Institute for Plasma Research, India

Ms. P.J. Pathak, Librarian, Insitute for Plasma Research, India

Ms. Clelia De Palo, Associazione EURATOM-ENEA, Italy

Dr. G. Grosso, Instituto di Fisica del Plasma, Italy

Librarian, Naka Fusion Research Establishment, JAERI, Japan

Library, Plasma Physics Laboratory, Kyoto University, Japan

Research Information Center, National Institute for Fusion Science, Japan

Dr. O. Mitarai, Kyushu Tokai University, Japan

Library, Academia Sinica, Institute of Plasma Physics, People's Republic of China

Shih-Tung Tsai, Institute of Physics, Chinese Academy of Sciences, People's Republic of China

Dr. S. Mirnov, TRINITI, Troitsk, Russian Federation, Russia

Dr. V.S. Strelkov, Kurchatov Institute, Russian Federation, Russia

Professor Peter Lukac, Katedra Fyziky Plazmy MFF UK, Mlynska dolina F-2, Komenskeho Univerzita, SK-842 15 Bratislava, Slovakia

Dr. G.S. Lee, Korea Basic Science Institute, South Korea

Institute for Plasma Research, University of Maryland, USA

Librarian, Fusion Energy Division, Oak Ridge National Laboratory, USA

Librarian, Institute of Fusion Studies, University of Texas, USA

Librarian, Magnetic Fusion Program, Lawrence Livermore National Laboratory, USA

Library, General Atomics, USA

Plasma Physics Group, Fusion Energy Research Program, University of California at San Diego, USA

Plasma Physics Library, Columbia University, USA

Alkesh Punjabi, Center for Fusion Research and Training, Hampton University, USA

Dr. W.M. Stacey, Fusion Research Center, Georgia Institute of Technology, USA

Dr. John Willis, U.S. Department of Energy, Office of Fusion Energy Sciences, USA

Mr. Paul H. Wright, Indianapolis, Indiana, USA 
The Princeton Plasma Physics Laboratory is operated by Princeton University under contract with the U.S. Department of Energy.

\author{
Information Services \\ Princeton Plasma Physics Laboratory \\ P.O. Box 451 \\ Princeton, NJ 08543
}

Phone: 609-243-2750

Fax: 609-243-2751

e-mail: pppl_info@pppl.gov

Internet Address: http://www.pppl.gov 